\title{
Coronary Arterial Remodeling and Out-Stent Plaque Change After Drug-Eluting Stent Implantation
}

\author{
- Comparison Between Zotarolimus-Eluting \\ Stents and Paclitaxel-Eluting Stents -
}

\author{
Yoshitaka Muraoka, MD, PhD; Shinjo Sonoda, MD, PhD; Kuninobu Kashiyama, MD; \\ Fumihiko Kamezaki, MD, PhD; Yuki Tsuda, MD, PhD; Masaru Araki, MD, PhD; \\ Masahito Tamura, MD, PhD; Masaaki Takeuchi, MD, PhD; Haruhiko Abe, MD, PhD; \\ Masahiro Okazaki, MD, PhD; Yutaka Otsuji, MD, PhD
}

\begin{abstract}
Background: Out-stent plaque characteristics and eosinophilic inflammatory response, which correlates with positive remodeling after first-generation drug-eluting stent implantation, may be associated with late restenosis and very late stent thrombosis. The differences of out-stent plaque characteristics were compared between paclitaxeleluting stents (PES) and zotarolimus-eluting stents (ZES), using integrated backscatter-intravascular ultrasound (IB-IVUS).
\end{abstract}

\begin{abstract}
Methods and Results: Of 78 patients enrolled, 25 receiving PES and 25 receiving ZES had adequate IVUS assessment. Volumetric IVUS analysis was performed after stenting and at 8-month follow-up. Out-stent plaque change in the stented segment was compared on IB-IVUS. The relationship between systemic inflammatory response and out-stent plaque change was evaluated. In PES, vessel volume significantly increased $\left(365-389 \mathrm{~mm}^{3}\right.$, $\mathrm{P}<0.0001)$, whereas it did not change in ZES (315-314 $\left.\mathrm{mm}^{3}, \mathrm{P}=0.81\right)$. In culprit lesions at baseline in PES, fibrous plaque tended to increase $\left(3.1-3.6 \mathrm{~mm}^{2}, \mathrm{P}=0.051\right)$ and lipid plaque significantly increased $\left(4.3-5.1 \mathrm{~mm}^{2}, \mathrm{P}=0.02\right)$, whereas in ZES the fibrous plaque significantly increased $\left(2.9-4.0 \mathrm{~mm}^{2}, \mathrm{P}<0.0001\right)$ but lipid plaque significantly decreased $\left(5.1-3.6 \mathrm{~mm}^{2}, \mathrm{P}<0.0001\right)$. Systemic eosinophil increase was significantly correlated with positive remodeling and out-stent lipid plaque increase.
\end{abstract}

Conclusions: Chronic out-stent plaque change in ZES consisted of less positive remodeling and more favorable effects on out-stent plaque characteristics than PES. Systemic eosinophil change might be a marker of out-stent lipid plaque change. (Circ J 2013; 77: 363-371)

Key Words: Drug-eluting stent; Intravascular ultrasound; Out-stent plaque; Paclitaxel-eluting stent; Zotarolimuseluting stent

$\mathbf{F}$ irst-generation polymer-based sirolimus (Cypher) and paclitaxel (Taxus) drug-eluting stents (DES) have remarkably reduced angiographic restenosis and target lesion revascularization (TLR) by reducing neointimal hyperplasia compared with bare-metal stents (BMS) ${ }^{1,2}$ Concern about vasculo-toxic effects, however, remains to be resolved. Previous case reports and observational studies showed that late and very late stent thrombosis occurred after DES implantation. $^{3,4}$

As the main mechanisms of late and very late stent thrombosis, delayed arterial healing (persistent fibrin deposition and delayed re-endothelialization) due to drugs in DES, ${ }^{5-9}$ and chronic inflammation and hypersensitivity reaction due to drugs and/or durable polymers in DES have been reported in animal and human pathology studies. ${ }^{7-12}$ These studies noted enhanced inflammatory responses consisting predominantly of $\mathrm{T}$ lymphocytes and eosinophils around the stent struts and stent malapposition with vessel enlargement after DES implantation. ${ }^{7,10-12}$ In clinical practice, previous intravascular ultrasound (IVUS) in vivo showed that positive coronary arterial remodeling after first-generation DES was associated with late restenosis and very late stent thrombosis. Those studies reported that positive

Received May 1, 2012; revised manuscript received August 26, 2012; accepted September 27, 2012; released online October 27, 2012 Time for primary review: 27 days

Second Department of Internal Medicine, University of Occupational and Environmental Health, School of Medicine, Kitakyushu, Japan Mailing address: Shinjo Sonoda, MD, PhD, Second Department of Internal Medicine, University of Occupational and Environmental Health, School of Medicine, 1-1 Iseigaoka, Yahatanishi-ku, Kitakyushu 807-8555, Japan. E-mail: s-sonoda@med.uoeh-u.ac.jp

ISSN-1346-9843 doi:10.1253/circj.CJ-12-0575

All rights are reserved to the Japanese Circulation Society. For permissions, please e-mail: cj@j-circ.or.jp 
vessel remodeling was the main cause of late-acquired incomplete stent apposition (ISA). It was also reported that lateacquired ISA seemed to be associated with late and very late stent thrombosis. ${ }^{13-18}$ Moreover, previous histopathological and IVUS studies showed that eosinophilic infiltrates were associated with evidence of vessel remodeling with late-acquired ISA. ${ }^{10,17}$

Endeavor $^{\mathrm{TM}}$ zotarolimus-eluting stents (ZES; Medtronic Vascular, Santa Rosa, CA, USA), which belong to secondgeneration DES, have biocompatible characteristics, with cobalt alloy thin-strut stent, phosphorylcholine polymer, and different drug-elution, compared with first-generation DES. ${ }^{19}$ Several past randomized trials confirmed the relative safety and efficacy of ZES compared with first-generation DES. ${ }^{19-21}$ IVUS in the Endeavor trials showed that ZES implantation provided relatively complete and uniform neointimal coverage over stent surface, resulting in the low incidence of late-acquired ISA.22-24 IVUS analysis from Endeavor IV trial showed that paclitaxel-eluting stents (PES) had significantly increased vessel and out-stent plaque volume (plaque behind the stent struts) in the stented segment during the follow-up period, whereas ZES had no significant changes in vessel or out-stent plaque volume. ${ }^{25}$ Little is known, however, about the tissue characteristics of chronic out-stent plaque after ZES implantation in vivo. We hypothesized that chronic out-stent plaque change in ZES would have a smaller systemic inflammatory response, accompanied by less positive vessel remodeling and more favorable effects on out-stent plaque characteristics than PES. Recently, integrated backscatter-IVUS (IB-IVUS) has enabled the in vivo tissue characterization of coronary plaque, such as calcification, dense fibrosis, fibrosis, and lipid, using radiofrequency ultrasound backscatter signals..$^{26-31}$ Therefore, we evaluated serial out-stent plaque characteristics after ZES implantation compared with PES implantation, using gray-scale IVUS and IB-IVUS. We also evaluated the correlation between serial systemic inflammatory response and out-stent plaque characteristics.

\section{Methods}

\section{Subjects}

From May 2009 to March 2010, after excluding patients with acute coronary syndrome, large vessel disease or low compliance with antiplatelet therapy (who were basically BMS candidates), we enrolled 78 consecutive patients with de novo lesions who underwent DES implantation. They were randomly assigned to be treated with PES ( $n=39)$ or ZES ( $n=39)$. Randomization was performed after diagnostic angiography and before percutaneous coronary intervention (PCI). Sequentially numbered, sealed randomization envelopes were used in accordance with a computer-generated random allocation sequence. Patients were randomized on a 1:1 basis to treatment with PES or ZES. Serial coronary angiography (CAG), grayscale IVUS and IB-IVUS were performed at baseline and at 8-month follow-up. Blood samples including representative systemic inflammation scores (white blood cell fraction and high-sensitivity C-reactive protein) were similarly collected. Patients with left main trunk disease, in-stent restenosis, and severe calcification lesions at follow-up catheterization were excluded from IVUS analysis. The patients with inadequate IVUS and IB-IVUS imaging due to lack of follow-up CAG and/or the IVUS catheter not crossing the culprit lesion or mechanical trouble were also excluded. The study was approved by the institutional review board. Consecutive and eligible patients signed written informed consent before the in- terventional procedure.

\section{Quantitative CAG}

CAG at baseline, immediately after PCI, and at follow-up was performed in at least 2 orthogonal views to avoid vessel overlapping after i.c. isosorbide dinitrate 1-2 mg. Quantitative CAG (QCA) was performed using an offline QCA system (CAAS QCA for Research2.0, Pie Medical Imaging BV, The Netherlands). The minimal luminal diameter of treated coronary segments, and the reference diameter on the baseline angiogram were determined in the view that demonstrated the lesion to be the most severe and not foreshortened. Then, minimal lumen diameter was measured on final angiogram. Minimal luminal diameter and late lumen loss were measured on follow-up angiogram.

\section{Acquisition of Gray-Scale IVUS and IB-IVUS Data}

All gray-scale IVUS and IB-IVUS was performed after i.c. isosorbide dinitrate 1-2 mg. Gray-scale IVUS and ultrasound signals were acquired with a commercially available IVUS imaging system (VISIWAVE, Terumo, Tokyo, Japan) using a 43-MHz mechanically rotating IVUS catheter (View IT, Terumo) with a motorized transducer pullback speed of $0.5 \mathrm{~mm} / \mathrm{s}$. During IVUS acquisition, radiofrequency signal, signal trigger, and video image output were automatically exported to a computer equipped with the custom software (IB-IVUS, YD, Nara, Japan) connected to the IVUS system. IB values for each tissue component were calculated as the average power using a fast Fourier transform, measured in decibels $(\mathrm{dB})$, of the frequency component of the backscattered signals from a small volume of tissue. IB-IVUS was also acquired at the interval of 1 image/s using an auto-pullback device $(0.5 \mathrm{~mm} / \mathrm{s})$. The excellent correlation of IB-IVUS and histology has been reported in the validation studies using not only a previous IVUS imaging system (Galaxy, Boston Scientific/Scimed, Natick, MA, USA) but also a new IVUS imaging system (VISIWAVE, Terumo). ${ }^{27,29}$ After tracing vessel, out-stent plaque, and stent area, grayscale IVUS images and IB-IVUS color-coded maps were displayed side-by-side on a monitor. Out-stent plaque tissue components were classified into 4 categories: calcification (red), dense fibrosis (yellow), fibrosis (green), and lipid (blue) according to signal level. The range of IB values for each historical category were the same as reported previously:29 -11 to $-20 \mathrm{~dB}$, calcification; -20 to $-26 \mathrm{~dB}$, dense fibrosis; -26 to $-42 \mathrm{~dB}$, fibrosis; and -42 to $-130 \mathrm{~dB}$, lipid.

\section{Gray-Scale IVUS and IB-IVUS Parameters}

On gray-scale IVUS analysis, cross-sectional images were quantified for stent, vessel, and out-stent plaque areas. Out-stent plaque area was calculated as vessel minus stent area. The percentages of calcification area (calcification area/out-stent plaque area), dense fibrosis area (dense fibrosis area/out-stent plaque area), fibrosis area (fibrosis area/out-stent plaque area), and lipid area (lipid area/out-stent plaque area) were automatically calculated by the IB-IVUS system. Serial quantitative IVUS was performed at baseline and at 8-month follow-up. Baseline and follow-up IVUS and IB-IVUS images were matched by the distances from stent edges. Because stent struts cause artifacts, we excluded the image frames including total shadowing due to struts, from analysis. After cross-sectional analysis was performed in culprit lesion at baseline, volumetric analysis was performed in the whole stent segment at 1-mm intervals. Vascular remodeling was assessed according to the change in vessel size (follow-up vessel area/baseline vessel area, follow-up vessel volume/baseline vessel volume). Posi- 


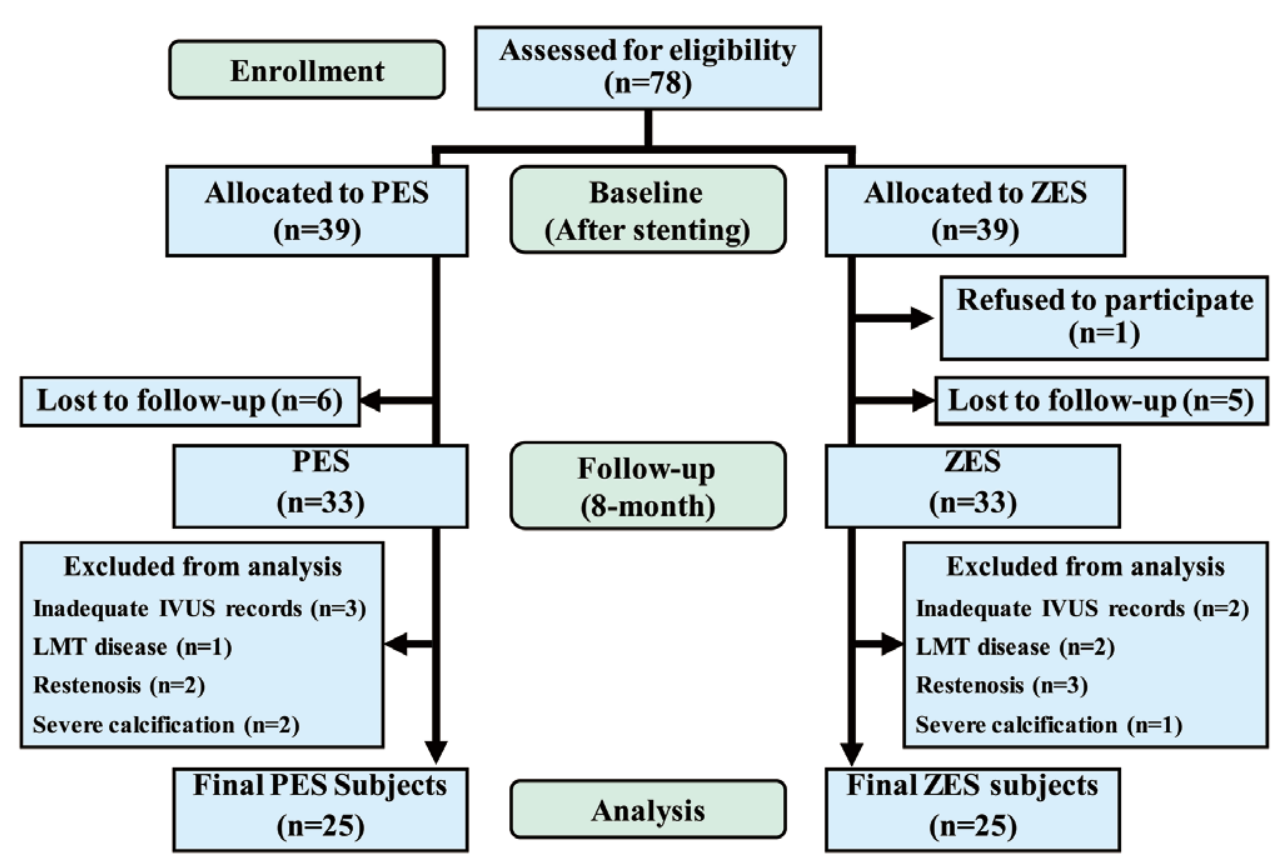

Figure 1. Flow of participants through the study. IVUS, intravascular ultrasound; LMT, left main trunk disease; PES, paclitaxeleluting stent; ZES, zotarolimus-eluting stent.

tive vessel remodeling was defined as a 5\% increase in vessel size. ${ }^{32}$ ISA was assessed on qualitative IVUS. ISA was defined as one or more struts clearly separated from the vessel wall with evidence of blood speckles behind the strut in a vessel segment not associated with any side branches. ISA was classified as persistent, resolved, or late-acquired as previously reported..$^{13}$ We also evaluated the correlation between serial change of systemic inflammation scores and out-stent plaque characteristics. All images were reviewed by 2 independent observers (S.S., K.K.), and adjudication of opinion was based on the consensus of these observers.

\section{Study Endpoints}

The primary endpoint was out-stent plaque volume and characteristics change after DES implantation. The secondary endpoint was serial systemic inflammatory response after DES implantation. We also evaluated major adverse cardiac events: death from cardiac causes, myocardial infarction, and TLR. TLR was defined as a repeated revascularization, based on a stenosis within the stent or within the 5-mm borders proximal or distal to the stent. TLR was considered if the stenosis of the target lesion was $>50 \%$ based on QCA in the presence of ischemic signs or symptoms, or if there was a stenosis of $>75 \%$ even in the absence of ischemic signs or symptoms.

\section{Statistical Analysis}

Continuous variables are expressed as mean \pm SD. Categorical data were compared using chi-squared statistic or Fisher's exact test. For continuous variables, comparisons between ZES and PES were performed with a 2-tailed, unpaired t-test, and comparisons between baseline and follow-up were done with a 2-tailed, paired t-test. Correlation between out-stent plaque change and systemic inflammation scores was analyzed using
Pearson's correlation analysis. $\mathrm{P}<0.05$ was considered statistically significant.

\section{Results}

Figure 1 shows the flow of participants through the study. Complete IVUS analysis was obtained in 25 patients receiving $\mathrm{PES}$ and in 25 patients receiving ZES.

Baseline clinical characteristics are summarized in Table $\mathbf{1 .}$ There were no significant differences in age, sex, coronary risk factors, or medication between the 2 groups. CAG findings and procedural results are listed in Table 2. More lesions in the left anterior descending artery were treated in ZES, although it was not significant. As for procedure characteristics, there were no significant differences in stent number, diameter, or length between groups. High pressure post-dilatation using non-compliant balloon was performed in both groups. Regarding QCA analysis, all parameters were similar at baseline and follow-up between the 2 groups. There was no cardiac death or myocardial infarction in either group. TLR rate was lower in the ZES group compared with PES, although it was not significant $(\mathrm{P}=0.46)$.

Serial changes of laboratory data are listed in Table 3. Absolute eosinophils significantly decreased at follow-up in ZES. There were no significant serial changes in other systemic inflammatory scores in either group. Serial eosinophil change, however, was not advanced and largely within the normal range.

\section{IVUS and IB-IVUS}

Serial gray-scale IVUS measurements are listed in Table 4. In the overall analysis, baseline IVUS measurements were not significantly different between groups. In cross-sectional and 


\begin{tabular}{|lrcc|}
\hline \multicolumn{3}{l}{ Table 1. Baseline Clinical Characteristics } \\
Clinical & PES & ZES & P-value \\
Age (years) & & & \\
$\quad$ Gender, male & $69 \pm 11$ & $70 \pm 10$ & 0.73 \\
Coronary risk factors & $18(72)$ & $15(60)$ & 0.37 \\
Hypertension & & & \\
Dyslipidemia & $21(84)$ & $16(64)$ & 0.20 \\
Diabetes mellitus & $18(72)$ & $17(68)$ & 0.76 \\
Smoking & $18(72)$ & $14(56)$ & 0.24 \\
Hemodialysis & $17(68)$ & $11(44)$ & 0.09 \\
Previous MI & $7(28)$ & $3(12)$ & 0.29 \\
Prior PCl or CABG & $7(28)$ & $6(24)$ & 0.75 \\
Family history & $13(52)$ & $11(44)$ & 0.57 \\
Medication & $6(24)$ & $2(8)$ & 0.25 \\
Dual antiplatelet therapy & $25(100)$ & $25(100)$ & - \\
ACEl/ARB & $19(76)$ & $13(52)$ & 0.08 \\
$\beta$-blocker & $10(40)$ & $6(24)$ & 0.23 \\
Ca channel blocker & $13(52)$ & $11(44)$ & 0.57 \\
Nicorandil & $7(28)$ & $10(40)$ & 0.37 \\
Nitrates & $4(16)$ & $5(20)$ & 0.99 \\
Statins & $22(88)$ & $22(88)$ & 0.99 \\
\hline
\end{tabular}

Data given as $\mathrm{n}(\%)$ or mean $\pm \mathrm{SD}$.

$\mathrm{ACEI}$, angiotensin-converting enzyme inhibitor; $\mathrm{ARB}$, angiotensin receptor blocker; CABG, coronary artery bypass grafting; $\mathrm{MI}$, myocardial infarction; $\mathrm{PCl}$, percutaneous coronary intervention; PES, paclitaxel-eluting stent; ZES, zotarolimus-eluting stent.

\begin{tabular}{|lrcc|}
\hline \multicolumn{4}{|c|}{ Table 2. Coronary Angiography and Procedure Results } \\
Target vessel & PES & ZES & P-value \\
LAD & & & 0.06 \\
LCX & $11(44)$ & $18(72)$ & \\
RCA & $3(12)$ & $0(0)$ & \\
ACC/AHA lesion type & $11(44)$ & $7(28)$ & \\
A/B1 & & & 0.51 \\
B2/C & $5(20)$ & $7(28)$ & \\
Procedural characteristics & $20(80)$ & $18(72)$ & \\
Stent number & & & \\
Use of multiple stents & $1.2 \pm 0.4$ & $1.2 \pm 0.4$ & 0.74 \\
Stent diameter (mm) & $6.1 \pm 0.3$ & $3.1 \pm 0.2$ & 0.75 \\
Stent length (mm) & $30.6 \pm 11.3$ & $27.7 \pm 10.0$ & 0.34 \\
Maximum pressure (atm) & $20 \pm 3$ & $21 \pm 2$ & 0.10 \\
QCA results & & & \\
Reference diameter (mm) & $2.1 \pm 0.5$ & $2.3 \pm 0.4$ & 0.31 \\
Minimal lumen diameter & & & \\
(mm) & & & \\
$\quad$ Before intervention & $0.7 \pm 0.3$ & $0.7 \pm 0.2$ & 0.73 \\
After intervention & $2.2 \pm 0.4$ & $2.3 \pm 0.4$ & 0.53 \\
8-month follow-up & $1.5 \pm 0.6$ & $1.6 \pm 0.5$ & 0.28 \\
Late lumen loss (mm) & $0.7 \pm 0.5$ & $0.6 \pm 0.5$ & 0.55 \\
Binary restenosis & $6(24)$ & $4(16)$ & 0.73 \\
TLR & $6(24)$ & $3(12)$ & 0.46 \\
\hline
\end{tabular}

Data given as $\mathrm{n}(\%)$ or mean $\pm \mathrm{SD}$.

$L A D$, left anterior descending; LCX, left circumflex; $Q C A$, quantitative coronary angiography; RCA, right coronary artery; TLR, target lesion revascularization. Other abbreviations as in Table 1.

\begin{tabular}{|lcc|}
\hline \multicolumn{2}{|c|}{ Table 3. Laboratory Data (Serial Change) } \\
WBC & Baseline & Follow-up \\
PES & $7,308 \pm 2,512$ & $6,644 \pm 2,092$ \\
ZES & $5,844 \pm 1,741$ & $5,800 \pm 1,818$ \\
Neutrophils $\left(/ \mathrm{mm}^{3}\right)$ & & \\
PES & $5,027 \pm 2,186$ & $4,413 \pm 1,572$ \\
ZES & $3,630 \pm 1,356$ & $3,714 \pm 1,406$ \\
Eosinophils $\left(/ \mathrm{mm}^{3}\right)$ & & \\
PES & $186 \pm 137$ & $201 \pm 151$ \\
ZES & $223 \pm 153$ & $169 \pm 145^{\star}$ \\
Monocytes $\left(/ \mathrm{mm}^{3}\right)$ & & \\
PES & $400 \pm 220$ & $345 \pm 126$ \\
ZES & $365 \pm 102$ & $352 \pm 131$ \\
hsCRP & & \\
PES & $0.6 \pm 1.7$ & $0.2 \pm 0.2$ \\
ZES & $0.5 \pm 0.6$ & $0.3 \pm 0.6$ \\
\hline
\end{tabular}

Data given as mean $\pm S D$. ${ }^{*} P<0.05$, follow-up vs. baseline. hsCRP, high-sensitivity C-reactive protein; WBC, white blood cells. Other abbreviations as in Table 1.

volumetric analysis, vessel size was significantly augmented in PES with significant increase in out-stent plaque $\left(17.5 \pm 4.2 \mathrm{~mm}^{2}\right.$ to $19.0 \pm 4.5 \mathrm{~mm}^{2}, \mathrm{P}<0.0001 ; 365 \pm 234 \mathrm{~mm}^{3}$ to $389 \pm 247 \mathrm{~mm}^{3}$, $\mathrm{P}<0.0001)$, whereas it did not change in ZES $\left(18.3 \pm 3.6 \mathrm{~mm}^{2}\right.$ to $18.2 \pm 3.6 \mathrm{~mm}^{2}, \mathrm{P}=0.49 ; 315 \pm 120 \mathrm{~mm}^{3}$ to $314 \pm 119 \mathrm{~mm}^{3}$, $\mathrm{P}=0.81$ ). Serial change in out-stent plaque was significantly larger in PES than in ZES $\left(+1.5 \pm 1.4 \mathrm{~mm}^{2}\right.$ vs. $-0.1 \pm 0.6 \mathrm{~mm}^{2}$, $\mathrm{P}<0.0001 ; 24.2 \pm 23.5 \mathrm{~mm}^{3}$ vs. $-0.4 \pm 8.9 \mathrm{~mm}^{3}, \mathrm{P}<0.0001$ ). Remodeling index was significantly larger in PES than in ZES (vessel area, $1.09 \pm 0.08$ vs. $0.99 \pm 0.03, \mathrm{P}<0.0001$; vessel volume, $1.06 \pm 0.05$ vs. $0.99 \pm 0.03, P<0.0001$ ). The incidence of positive vessel remodeling was significantly higher in PES than in ZES $(56 \%, \mathrm{n}=14$ vs. $4 \%, \mathrm{n}=1 ; \mathrm{P}=0.0001$ for both vessel area and volume). On qualitative IVUS, there were 2 ISA at baseline in PES and 4 ISA at baseline in ZES. Although most ISA at baseline resolved at 8-month follow-up, there were 2 late cases of ISA at follow-up in PES, whereas there were no such cases in ZES. Representative 2-D IB-IVUS color-coded maps of out-stent plaque in culprit site in each group are given in Figure 2. Serial IB-IVUS measurements are given in Table 5. In PES, fibrous plaque tended to or significantly increased $\left(3.1 \pm 1.2 \mathrm{~mm}^{2}\right.$ to $3.6 \pm 1.0 \mathrm{~mm}^{2}, \mathrm{P}=0.051 ; 56.4 \pm 34.5 \mathrm{~mm}^{3}$ to $70.3 \pm 39.7 \mathrm{~mm}^{3}, \mathrm{P}=0.0011$ ) and lipid plaque tended to or significantly increased $\left(4.3 \pm 3.0 \mathrm{~mm}^{2}\right.$ to $5.1 \pm 3.2 \mathrm{~mm}^{2}, \mathrm{P}=0.02$; $85.3 \pm 90.0 \mathrm{~mm}^{3}$ to $91.2 \pm 97.6 \mathrm{~mm}^{3}, \mathrm{P}=0.12$ ), whereas fibrous plaque significantly increased $\left(2.9 \pm 0.5 \mathrm{~mm}^{2}\right.$ to $4.0 \pm 1.2 \mathrm{~mm}^{2}$, $\mathrm{P}<0.0001 ; 54.1 \pm 21.2 \mathrm{~mm}^{3}$ to $\left.67.4 \pm 27.7 \mathrm{~mm}^{3}, \mathrm{P}<0.0001\right)$ and lipid plaque significantly decreased $\left(5.1 \pm 2.9 \mathrm{~mm}^{2}\right.$ to $3.6 \pm 2.8 \mathrm{~mm}^{2}$, $\mathrm{P}<0.0001 ; 65.0 \pm 41.4 \mathrm{~mm}^{3}$ to $47.5 \pm 35.9 \mathrm{~mm}^{3}, \mathrm{P}=0.0001$ ) in ZES. Serial lipid plaque change showed increased response in PES, while it showed decreased response in ZES $\left(0.8 \pm 1.6 \mathrm{~mm}^{2}\right.$ vs. $-1.5 \pm 1.1 \mathrm{~mm}^{2}, \mathrm{P}<0.0001 ; 5.9 \pm 18.3 \mathrm{~mm}^{3}$ vs. $-17.5 \pm 18.8 \mathrm{~mm}^{3}$, $\mathrm{P}<0.0001$ for both vessel area and volume).

In addition, we examined the relationship between systemic eosinophil change and out-stent plaque change, although fluctuations in eosinophil number after PES or ZES implantation were not advanced and largely within the normal range. Increase in eosinophils was significantly correlated with positive vessel remodeling $(\mathrm{r}=0.45, \mathrm{P}=0.001)$ and increase in out-stent lipid plaque ( $\mathrm{r}=0.47, \mathrm{P}=0.0006)$ at follow-up (Figure 3 ). There 


\begin{tabular}{|c|c|c|c|c|}
\hline \multirow[b]{3}{*}{ Culprit site } & \multicolumn{2}{|c|}{ PES } & \multicolumn{2}{|c|}{ ZES } \\
\hline & Mean \pm SD & FU-BL & Mean \pm SD & FU-BL \\
\hline & & & & \\
\hline \multicolumn{5}{|l|}{ Vessel area $\left(\mathrm{mm}^{2}\right)$} \\
\hline Baseline & $17.5 \pm 4.2$ & \multirow{2}{*}{$1.5 \pm 1.4^{\dagger}$} & $18.3 \pm 3.6$ & \multirow{2}{*}{$-0.1 \pm 0.6$} \\
\hline Follow-up & $19.0 \pm 4.5^{*}$ & & $18.2 \pm 3.6$ & \\
\hline Remodeling index & $1.09 \pm 0.08^{\dagger}$ & & $0.99 \pm 0.03$ & \\
\hline \multicolumn{5}{|c|}{ Out-stent plaque area $\left(\mathrm{mm}^{2}\right)$} \\
\hline Baseline & $7.9 \pm 3.2$ & \multirow{2}{*}{$1.5 \pm 1.3^{\dagger}$} & $8.2 \pm 2.9$ & \multirow{2}{*}{$-0.1 \pm 0.6$} \\
\hline Follow-up & $9.4 \pm 3.3^{\star}$ & & $8.2 \pm 3.0$ & \\
\hline \multicolumn{5}{|l|}{ Stent area $\left(\mathrm{mm}^{2}\right)$} \\
\hline Baseline & $9.7 \pm 2.3$ & \multirow{2}{*}{$0.01 \pm 0.5$} & $10.1 \pm 1.6$ & \multirow{2}{*}{$-0.01 \pm 0.4$} \\
\hline Follow-up & $9.7 \pm 2.3$ & & $10.1 \pm 1.7$ & \\
\hline \multicolumn{5}{|l|}{ Whole stent segment } \\
\hline \multicolumn{5}{|c|}{ Vessel volume $\left(\mathrm{mm}^{3}\right)$} \\
\hline Baseline & $365 \pm 234$ & \multirow{2}{*}{$24.2 \pm 23.5^{\dagger}$} & $315 \pm 120$ & \multirow{2}{*}{$-0.4 \pm 8.9$} \\
\hline Follow-up & $389 \pm 247^{*}$ & & $314 \pm 119$ & \\
\hline Remodeling index & $1.06 \pm 0.05^{\dagger}$ & & $0.99 \pm 0.03$ & \\
\hline \multicolumn{5}{|c|}{ Out-stent plaque volume $\left(\mathrm{mm}^{3}\right)$} \\
\hline Baseline & $152 \pm 125$ & \multirow{2}{*}{$22.7 \pm 18.1^{\dagger}$} & $128 \pm 60$ & \multirow{2}{*}{$0.05 \pm 8.4$} \\
\hline Follow-up & $174 \pm 135^{*}$ & & $128 \pm 58$ & \\
\hline \multicolumn{5}{|l|}{ Stent volume $\left(\mathrm{mm}^{3}\right)$} \\
\hline Baseline & $213 \pm 121$ & \multirow{2}{*}{$1.5 \pm 9.3$} & $187 \pm 69$ & \multirow{2}{*}{$-0.7 \pm 7.5$} \\
\hline Follow-up & $215 \pm 122$ & & $186 \pm 69$ & \\
\hline
\end{tabular}

${ }^{*} \mathrm{P}<0.0001, \mathrm{FU}$ vs. $\mathrm{BL} ;{ }^{\dagger} \mathrm{P}<0.0001, \mathrm{PES}$ vs. ZES.

BL, baseline; FU, follow-up; IVUS, intravascular ultrasound. Other abbreviations as in Table 1.

\section{A Baseline}



B

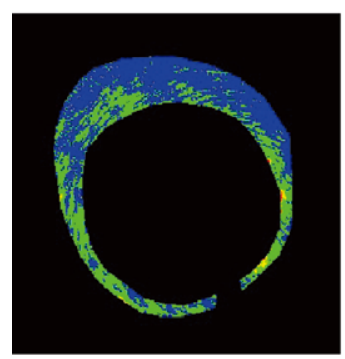

Vessel area: $21.3 \mathrm{~mm}^{2}$ Peristent area: $7.8 \mathrm{~mm}^{2}$ Stent area:

\begin{tabular}{|l|l|}
\hline & $0.01 \mathrm{~mm}^{2}$ \\
\hline & $0.07 \mathrm{~mm}^{2}$ \\
\hline & $3.15 \mathrm{~mm}^{2}$ \\
\hline & $4.57 \mathrm{~mm}^{2}$ \\
\hline
\end{tabular}

\section{Follow-up}

Vessel area: $21.2 \mathrm{~mm}^{2} \longrightarrow 22.9 \mathrm{~mm}^{2}$ Peristent area: $9.9 \mathrm{~mm}^{2} \longrightarrow 11.5 \mathrm{~mm}^{2}$ Stent area: $\quad 11.3 \mathrm{~mm}^{2}$

\begin{tabular}{|l|l|}
\hline & $0.03 \mathrm{~mm}^{2}$ \\
\hline $0.20 \mathrm{~mm}^{2}$ \\
\hline $6.57 \mathrm{~mm}^{2}$ \\
\hline $3.10 \mathrm{~mm}^{2}$ \\
\hline
\end{tabular}
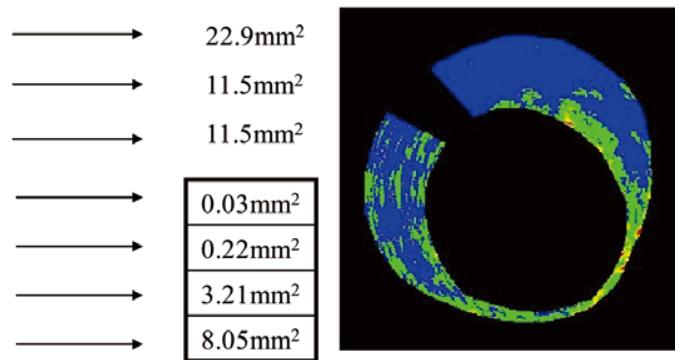

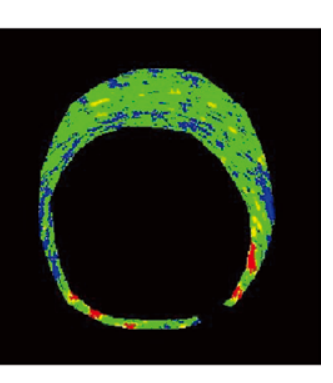

Figure 2. Representative 2-D integrated backscatter-intravascular ultrasound color-coded maps of out-stent plaque at the culprit site at baseline for (A) paclitaxel-eluting stent (PES) and (B) zotarolimus-eluting stent (ZES). Red, calcification; yellow, dense fibrosis; green, fibrosis; blue, lipid. Each out-stent plaque component is described as a percentage. Fibrous plaque apparently decreased but lipid plaque apparently increased in PES, whereas fibrous plaque apparently increased but lipid plaque apparently decreased in ZES, at follow-up. 


\begin{tabular}{|c|c|c|c|c|}
\hline & \multicolumn{2}{|c|}{ PES } & \multicolumn{2}{|c|}{ ZES } \\
\hline & Mean \pm SD & FU-BL & Mean \pm SD & FU-BL \\
\hline \multicolumn{5}{|l|}{ Culprit site } \\
\hline \multicolumn{5}{|c|}{ Calcification area $\left(\mathrm{mm}^{2}\right)$} \\
\hline Baseline & $0.1 \pm 0.1$ & \multirow{2}{*}{$0.05 \pm 0.2$} & $0.1 \pm 0.1$ & \multirow{2}{*}{$0.08 \pm 0.2$} \\
\hline Follow-up & $0.2 \pm 0.2$ & & $0.2 \pm 0.1^{*}$ & \\
\hline \multicolumn{5}{|c|}{ Dense fibrosis area $\left(\mathrm{mm}^{2}\right)$} \\
\hline Baseline & $0.3 \pm 0.2$ & \multirow{2}{*}{$0.1 \pm 0.2$} & $0.3 \pm 0.2$ & \multirow{2}{*}{$0.2 \pm 0.3$} \\
\hline Follow-up & $0.4 \pm 0.3^{*}$ & & $0.5 \pm 0.2^{*}$ & \\
\hline \multicolumn{5}{|c|}{ Fibrosis area $\left(\mathrm{mm}^{2}\right)$} \\
\hline Baseline & $3.1 \pm 1.2$ & \multirow{2}{*}{$0.6 \pm 1.3$} & $2.9 \pm 0.5$ & \multirow{2}{*}{$1.1 \pm 1.0$} \\
\hline Follow-up & $3.6 \pm 1.0$ & & $4.0 \pm 1.2^{*}$ & \\
\hline \multicolumn{5}{|c|}{ Lipid area $\left(\mathrm{mm}^{2}\right)$} \\
\hline Baseline & $4.3 \pm 3.0$ & \multirow{2}{*}{$0.8 \pm 1.6^{\dagger}$} & $5.1 \pm 2.9$ & \multirow{2}{*}{$-1.5 \pm 1.1$} \\
\hline Follow-up & $5.1 \pm 3.2^{*}$ & & $3.6 \pm 2.8^{*}$ & \\
\hline \multicolumn{5}{|c|}{ Whole stent segment } \\
\hline \multicolumn{5}{|c|}{ Calcification volume $\left(\mathrm{mm}^{3}\right)$} \\
\hline Baseline & $3.0 \pm 1.9$ & \multirow{2}{*}{$0.9 \pm 1.9$} & $2.3 \pm 1.6$ & \multirow{2}{*}{$1.3 \pm 2.3$} \\
\hline Follow-up & $4.0 \pm 2.5^{\star}$ & & $3.6 \pm 2.5^{\star}$ & \\
\hline \multicolumn{5}{|c|}{ Dense fibrosis volume $\left(\mathrm{mm}^{3}\right)$} \\
\hline Baseline & $6.9 \pm 3.9$ & \multirow{2}{*}{$14.3 \pm 3.8$} & $6.4 \pm 3.4$ & \multirow{2}{*}{$18.7 \pm 4.3$} \\
\hline Follow-up & $8.9 \pm 4.7^{*}$ & & $9.3 \pm 4.9^{*}$ & \\
\hline \multicolumn{5}{|c|}{ Fibrosis volume $\left(\mathrm{mm}^{3}\right)$} \\
\hline Baseline & $56.4 \pm 34.5$ & \multirow{2}{*}{$13.9 \pm 18.7$} & $54.1 \pm 21.2$ & \multirow{2}{*}{$13.3 \pm 13.3$} \\
\hline Follow-up & $70.3 \pm 39.7^{*}$ & & $67.4 \pm 27.7^{*}$ & \\
\hline \multicolumn{5}{|c|}{ Lipid volume $\left(\mathrm{mm}^{3}\right)$} \\
\hline Baseline & $85.3 \pm 90.0$ & \multirow{2}{*}{$5.9 \pm 18.3^{\dagger}$} & $65.0 \pm 41.4$ & \multirow{2}{*}{$-17.5 \pm 18.8$} \\
\hline Follow-up & $91.2 \pm 97.6$ & & $47.5 \pm 35.9^{*}$ & \\
\hline
\end{tabular}

${ }^{*} \mathrm{P}<0.05$, FU vs. $B L ;{ }^{\top} \mathrm{P}<0.0001, \mathrm{PES}$ vs. ZES.

IB, integrated backscatter. Other abbreviations as in Tables 1,4 .

A

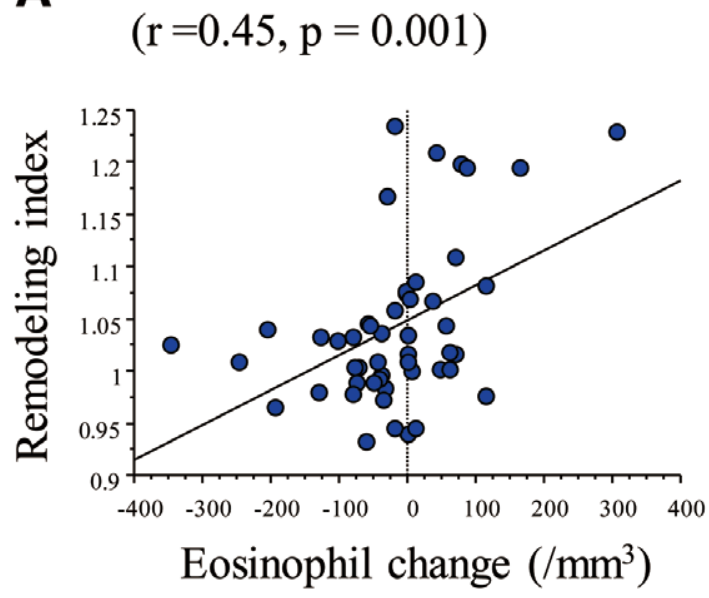

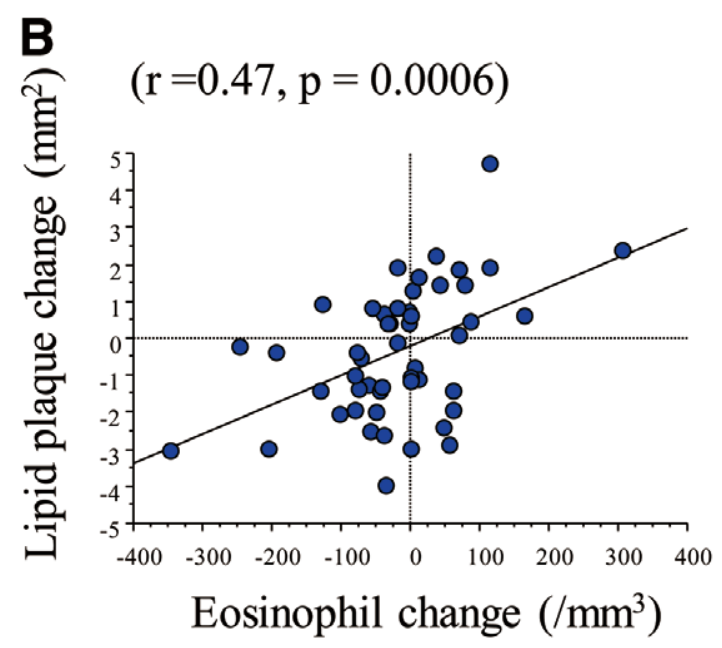

Figure 3. (A) Remodeling index vs. systemic eosinophil change. (B) Out-stent lipid plaque change vs. systemic eosinophil change. 
were no cases of late or very late stent thrombosis up to 30month follow-up in either group.

\section{Discussion}

The main results of this study are as follows. First, out-stent plaque change was different between PES and ZES. Serial gray-scale IVUS showed that vessel size significantly increased in PES, whereas it did not change in ZES on both culprit site and volumetric analysis within the stents. Remodeling index was significantly larger and the rate of positive vessel remodeling was significantly higher in PES than in ZES. Serial IBIVUS showed that fibrous plaque significantly increased and lipid plaque significantly increased in PES, whereas fibrous plaque significantly increased but lipid plaque significantly decreased in ZES, at the culprit site. And second, increase in eosinophils was significantly correlated with positive vessel remodeling and increase in out-stent lipid plaque at follow-up.

Although first-generation DES had dramatically reduced angiographic restenosis and TLR by decreasing neointimal hyperplasia compared with BMS,1,2 late and very late stent thrombosis, a life-threatening complication of this technology, has emerged as a major safety concern. ${ }^{3,4}$ As the main mechanisms of late and very late stent thrombosis, delayed arterial healing (persistent fibrin deposition and delayed re-endothelialization) due to drugs in DES ${ }^{5-9}$ and chronic inflammation and hypersensitive reactions due to drugs and/or durable polymers in DES, have been reported in animal and human pathological studies. ${ }^{7-12}$ These studies found enhanced inflammatory responses consisting predominantly of $\mathrm{T}$ lymphocytes and eosinophils around stent struts and stent malapposition with vessel enlargement after DES implantation. ${ }^{7,10-12}$ In clinical practice, previous in vivo IVUS showed that positive vessel remodeling after first-generation DES was associated with late and very late stent thrombosis. Those studies reported that positive vessel remodeling was the main cause of late-acquired ISA. It was also reported that late-acquired ISA seemed to correlate with late and very late stent thrombosis. ${ }^{13-18}$ Cook et al also reported that very late DES thrombosis was associated with histopathological signs of inflammation and IVUS evidence of vessel remodeling. Eosinophilic infiltrates were predominantly observed in thrombus aspirates, which suggested an immunologic reaction in response to the implanted DES. ${ }^{17}$ Furthermore, recent pathology suggested that neoatherosclerosis occurred in both DES and BMS, but it was observed more frequently and earlier in DES compared with BMS. That study also suggested that neoatherosclerosis could be accelerated in DES and, in rare cases, contributed to very late thrombotic events. ${ }^{33}$ In previous angioscopic clinical studies, DES showed poor neointimal coverage and promoted formation of yellow plaque in stented lesions at chronic phase. ${ }^{34,35}$

Endeavor ${ }^{\mathrm{TM}} \mathrm{ZES}$ (Medtronic Vascular), second-generation DES, have biocompatible characteristics with low-profile, thinstrut, cobalt-alloy Driver stent, phosphorylcholine polymer, and different drug-elution, compared with first-generation DES. ${ }^{19}$ According to several randomized trials, ZES was found to have relative safety and efficacy compared with first-generation DES. ${ }^{19-21}$ Previous optical coherence tomography and angioscopic clinical studies demonstrated that ZES provided adequate neointimal coverage and that late-acquired ISA was not found after stenting, which indicated arterial endothelial healing after stenting. ${ }^{36-38}$ Moreover, the angioscopic study reported that ZES-implanted vessels were associated with significantly less yellow plaques than first-generation DES. ${ }^{38}$ This finding suggests that ZES may provide stabilization of residual plaque.
IVUS analyses from Endeavor trials showed that ZES implantation provided relatively complete and uniform neointimal coverage over the stent surface, resulting in low incidence of late-acquired ISA. ${ }^{22-24}$ IVUS analysis from the Endeavor IV trial showed that PES had significantly increased vessel and out-stent plaque volume in stented segments during the followup period, whereas ZES had no significant changes in vessel and out-stent plaque volume. ${ }^{25}$

In the present study, QCA showed that there were no significant differences in any parameters or in the TLR rate between the 2 groups. Chronic out-stent plaque change after ZES implantation, however, was different from that after PES implantation. ZES had less positive vessel remodeling and more favorable changes in out-stent plaque characteristics (fibrous plaque increase and lipid plaque decrease) in comparison with PES. Owing to rapid drug-elution, much less chronic inflammation and allergy caused by durable polymers, sufficient neointimal coverage, composed mainly of smooth muscle cells and collagen fibers, can occur to seal the residual plaque behind stent struts. This suggests that favorable arterial healing will occur after stenting. Moreover, it is expected that favorable arterial healing may lead to the stabilization of residual plaque with less positive vessel remodeling. Out-stent fibrous plaque increase and lipid plaque decrease on IB-IVUS analysis may suggest that favorable arterial healing and stabilization of residual plaque will occur. In addition, almost all patients received statin treatment. Several previous studies of lipid-lowering therapy found that statin treatment significantly decreased atheromatous plaque volume and lipid components. ${ }^{39}$ These results are thought to be due to lipid-lowering action and pleiotropic effects. It is possible that statin treatment may have made some contribution to the stabilization of residual plaque after stent implantation in this study. In contrast, PES caused positive vessel remodeling and unfavorable changes in outstent plaque characteristics (lipid plaque increase). Because of delayed arterial healing due to drugs, chronic inflammation and hypersensitivity reaction caused by drugs and/or durable polymers, incomplete neointimal coverage, that is, a deficiency of smooth muscle cells and collagen fiber progression, and an abundance of vulnerable tissue, such as thrombus, fibrin, and proteoglycan can occur. Recent pathological and IVUS studies suggested that positive vessel remodeling after PES implantation was caused by vascular toxicity (tissue necrosis and apoptosis) due to excessive drug elution and hypersensitive reactions due to drugs and/or durable polymers. ${ }^{25,40,41}$ What is worse, it is possible that residual plaque can cause progression of atherosclerosis in the early phase. Increase of vessel size and out-stent lipid plaque on IVUS and IB-IVUS may suggest the presence of vulnerable tissue, such as thrombus, fibrin, and proteoglycan and the progression of atherosclerosis.

Regarding eosinophil change, because there could be multiple factors in the increase or decrease of eosinophils, eosinophils may increase after PES implantation in some cases, while eosinophils may decrease in others. Similar results were also observed after ZES implantation in this study. It is difficult to conclude that PES or ZES has a bidirectional influence on eosinophil number according to the present results. Although eosinophil number significantly decreased after ZES implantation, the change was not advanced and largely within the normal range. Possible reasons for the reduced eosinophils after ZES implantation are as follows: (1) a plaque-sealing effect of out-stent plaque inflammation due to favorable arterial healing and sufficient neointimal coverage; and (2) lipidlowering action and pleiotropic effects due to statin treatment. Further studies are necessary to evaluate this point. 
Despite these, we consider that eosinophil number fluctuations after PES or ZES implantation, although not advanced and largely within normal range, are important. Increase in eosinophils was significantly correlated with positive vessel remodeling and increase in out-stent lipid plaque at follow-up. There is a possibility that residual drug and/or durable polymers were associated with chronic inflammation and allergy. Statin treatment may not be able to produce favorable vessel response.

\section{Study Limitations}

This study had several limitations. It was a small randomized study performed at a single medical center, raising the possibility of selection bias. Although QCA and IVUS analysis were performed independently by 2 experienced blinded observers, there were big differences among QCA-derived reference diameter and post-intervention minimal lumen diameter, and actual stent diameter. In the previous IVUS study, the difference between QCA and IVUS was $>0.5 \mathrm{~mm}$ when reference diameter on angiography was $<2.5 \mathrm{~mm} .{ }^{42}$ In IVUS analysis, vessel measurement was performed only for lesions for which vessel borders were clearly visible. Lesions in which vessel borders were not clearly visible on IVUS (heavily calcified lesions) were excluded in this study. Large vessel size as well as existence of neointima and stent struts may affect the results of out-stent plaque characterization because of echo attenuation. We believe, however, that we conducted the measurements under the same conditions, that is, in exactly the same place and using the same method, so that we could minimize the effect of this. Moreover, the IB-IVUS system in itself can correct the effect of attenuation. ${ }^{29}$ Supportive evidence of chronic coronary arterial response after DES implantation in humans is mostly derived from autopsy studies. IVUS and IB-IVUS are the only systems that enable vessel and out-stent plaque change to be observed in vivo. ${ }^{43}$ Therefore, we evaluated the differences of vessel response between ZES and PES using IVUS and IB-IVUS. Validation on pathology is needed in the future. Because radiofrequency quality greatly deteriorates behind the stent, we excluded from analysis the image frames including total shadowing due to struts. It was difficult to distinguish between thrombus and other plaque components on IB-IVUS. Follow-up IVUS was limited to a mid-term period of 8 months. The impacts of vessel remodeling and outstent plaque change on clinical outcome are still unclear. Further studies with longer-term follow-up are necessary for more adequate assessment of these findings.

\section{Conclusions}

Chronic out-stent plaque change in ZES involved less positive vessel remodeling and more favorable effects on out-stent plaque characteristics than PES. Serial systemic eosinophil response largely in the normal range in PES might be associated with increase of vessel remodeling and out-stent lipid plaque. Further studies are required to confirm the clinical impact of these findings.

\section{Acknowledgment}

We have no financial or other relationships that could lead to conflict of interest.

\section{References}

1. Morice MC, Serruys PW, Sousa JE, Fajadet J, Ban Hayashi E, Perin $\mathrm{M}$, et al. A randomized comparison of a sirolimus-eluting stent with a standard stent for coronary revascularization. N Engl J Med 2002;
346: $1773-1780$.

2. Stone GW, Ellis SG, Cox DA, Hermiller J, O'Shaughnessy C, Mann JT, et al. A polymer-based, paclitaxel-eluting stent in patients with coronary artery disease. N Engl J Med 2004; 350: $221-231$.

3. McFadden EP, Stabile E, Regar E, Cheneau E, Ong AT, Kinnaird T, et al. Late thrombosis in drug-eluting coronary stents after discontinuation of antiplatelet therapy. Lancet 2004; 364: 1519-1521.

4. Daemen J, Wenaweser P, Tsuchida K, Abrecht L, Vaina S, Morger C, et al. Early and late coronary stent thrombosis of sirolimus-eluting and paclitaxel-eluting stents in routine clinical practice: Data from a large two-institutional cohort study. Lancet 2007; 369: 667-678.

5. Joner M, Finn AV, Farb A, Mont EK, Kolodgie FD, Ladich E, et al. Pathology of drug-eluting stents in humans: Delayed healing and late thrombotic risk. J Am Coll Cardiol 2006; 48: 193-202.

6. Finn AV, Joner M, Nakazawa G, Kolodgie F, Newell J, John MC, et al. Pathological correlates of late drug-eluting stent thrombosis: Strut coverage as a marker of endothelialization. Circulation 2007; 115: $2435-2441$

7. Finn AV, Nakazawa G, Joner M, Kolodgie FD, Mont EK, Gold HK, et al. Vascular responses to drug eluting stents: Importance of delayed healing. Arterioscler Thromb Vasc Biol 2007; 27: 1500-1510.

8. Nakazawa G, Finn AV, Joner M, Ladich E, Kutys R, Mont EK, et al. Delayed arterial healing and increased late stent thrombosis at culprit sites after drug-eluting stent placement for acute myocardial infarction patients: An autopsy study. Circulation 2008; 118: 1138-1145.

9. Hao H, Ishibashi-Ueda H, Tsujimoto M, Ueda Y, Shite J, Gabbiani G, et al. Drug-eluting stent: Importance of clinico-pathological correlations. Circ J 2011; 75: 1548-1558.

10. Virmani R, Guagliumi G, Farb A, Musumeci G, Grieco N, Motta T, et al. Localized hypersensitivity and late coronary thrombosis secondary to a sirolimus-eluting stent: Should we be cautious? Circulation 2004; 109: 701-705.

11. Nebeker JR, Virmani R, Bennett CL, Hoffman JM, Samore MH, Alvarez J, et al. Hypersensitivity cases associated with drug-eluting coronary stents: A review of available cases from the Research on Adverse Drug Events and Reports (RADAR) project. J Am Coll Cardiol 2006; 47: 175-181.

12. Nakazawa G, Finn AV, Vorpahl M, Ladich ER, Kolodgie FD, Virmani R. Coronary responses and differential mechanisms of late stent thrombosis attributed to first-generation sirolimus- and paclitaxel-eluting stents. J Am Coll Cardiol 2011; 57: 390-398.

13. Ako J, Morino Y, Honda Y, Hassan A, Sonoda S, Yock PG, et al. Late incomplete stent apposition after sirolimus-eluting stent implantation: A serial intravascular ultrasound analysis. J Am Coll Cardiol 2005; 46: $1002-1005$.

14. Tanabe K, Serruys PW, Degertekin M, Grube E, Guagliumi G, Urbaszek W, et al. Incomplete stent apposition after implantation of paclitaxel-eluting stents or bare metal stents: Insights from the randomized TAXUS II trial. Circulation 2005; 111: 900-905

15. Hong MK, Mintz GS, Lee CW, Park DW, Park KM, Lee BK, et al. Late stent malapposition after drug-eluting stent implantation: An intravascular ultrasound analysis with long-term follow-up. Circulation 2006; 113: 414-419.

16. Cook S, Wenaweser P, Togni M, Billinger M, Morger C, Seiler C, et al. Incomplete stent apposition and very late stent thrombosis after drug-eluting stent implantation. Circulation 2007; 115: 2426-2434.

17. Cook S, Ladich E, Nakazawa G, Eshtehardi P, Neidhart M, Vogel R, et al. Correlation of intravascular ultrasound findings with histopathological analysis of thrombus aspirates in patients with very late drugeluting stent thrombosis. Circulation 2009; 120: 391-399.

18. Hassan AK, Bergheanu SC, Stijnen T, van der Hoeven BL, Snoep JD, Plevier JW, et al. Late stent malapposition risk is higher after drugeluting stent compared with bare-metal stent implantation and associates with late stent thrombosis. Eur Heart J 2010; 31: 1172-1180.

19. Pinto Slottow TL, Waksman R. Overview of the 2007 Food and Drug Administration Circulatory System Devices Panel meeting on the endeavor zotarolimus-eluting coronary stent. Circulation 2008; 117: $1603-1608$.

20. Kandzari DE, Leon MB, Popma JJ, Fitzgerald PJ, O'Shaughnessy C, Ball MW, et al. Comparison of zotarolimus-eluting and sirolimuseluting stents in patients with native coronary artery disease: A randomized controlled trial. J Am Coll Cardiol 2006; 48: 2440-2447.

21. Park DW, Kim YH, Yun SC, Kang SJ, Lee SW, Lee CW, et al. Comparison of zotarolimus-eluting stents with sirolimus- and paclitaxeleluting stents for coronary revascularization: The ZEST (comparison of the efficacy and safety of zotarolimus-eluting stent with sirolimuseluting and paclitaxel-eluting stent for coronary lesions) randomized trial. J Am Coll Cardiol 2010; 56: 1187-1195.

22. Feres F, Andrade PB, Costa RA, de Ribamar Costa J Jr, Abizaid A, Staico R, et al. Angiographic and intravascular ultrasound findings 
following implantation of the Endeavor zotarolimus-eluting stents in patients from the real-world clinical practice. EuroIntervention 2009; 5: $355-362$.

23. Sakurai R, Bonneau HN, Honda Y, Fitzgerald PJ. Intravascular ultrasound findings in ENDEAVOR II and ENDEAVOR III. Am J Cardiol 2007; 100: 71M-76M.

24. Miyazawa A, Ako J, Hongo Y, Hur SH, Tsujino I, Courtney BK, et al. Comparison of vascular response to zotarolimus-eluting stent versus sirolimus-eluting stent: Intravascular ultrasound results from ENDEAVOR III. Am Heart J 2008; 155: 108-113.

25. Waseda K, Miyazawa A, Ako J, Hasegawa T, Tsujino I, Sakurai R, et al. Intravascular ultrasound results from the ENDEAVOR IV trial: Randomized comparison between zotarolimus- and paclitaxel-eluting stents in patients with coronary artery disease. JACC Cardiovasc Interv 2009; 2: 779-784.

26. Kawasaki M, Takatsu H, Noda T, Sano K, Ito Y, Hayakawa K, et al. In vivo quantitative tissue characterization of human coronary arterial plaques by use of integrated backscatter intravascular ultrasound and comparison with angioscopic findings. Circulation 2002; 105: $2487-2492$.

27. Okubo M, Kawasaki M, Ishihara Y, Takeyama U, Kubota T, Yamaki $\mathrm{T}$, et al. Development of integrated backscatter intravascular ultrasound for tissue characterization of coronary plaques. Ultrasound Med Biol 2008; 34: 655-663.

28. Okubo M, Kawasaki M, Ishihara Y, Takeyama U, Yasuda S, Kubota $\mathrm{T}$, et al. Tissue characterization of coronary plaques: Comparison of integrated backscatter intravascular ultrasound with virtual histology intravascular ultrasound. Circ J 2008; 72: 1631-1639.

29. Kawasaki M, Hattori A, Ishihara Y, Okubo M, Nishigaki K, Takemura $\mathrm{G}$, et al. Tissue characterization of coronary plaques and assessment of thickness of fibrous cap using integrated backscatter intravascular ultrasound. Comparison with histology and optical coherence tomography. Circ J 2010; 74: 2641-2648.

30. Amano T, Matsubara T, Uetani T, Kato M, Kato B, Yoshida T, et al. Impact of omega- 3 polyunsaturated fatty acids on coronary plaque instability: An integrated backscatter intravascular ultrasound study. Atherosclerosis 2011; 218: 110-116.

31. Ohota M, Kawasaki M, Ismail TF, Hattori K, Serruys PW, Ozaki Y. A histological and clinical comparison of new and conventional integrated backscatter intravascular ultrasound (IB-IVUS). Circ J 2012; 76: $1678-1686$.

32. Schoenhagen P, Ziada KM, Kapadia SR, Crowe TD, Nissen SE, Tuzcu EM. Extent and direction of arterial remodeling in stable versus unstable coronary syndromes: An intravascular ultrasound study. Circulation 2000; 101: 598-603.
33. Nakazawa G, Otsuka F, Nakano M, Vorpahl M, Yazdani SK, Ladich $\mathrm{E}$, et al. The pathology of neoatherosclerosis in human coronary implants bare-metal and drug-eluting stents. J Am Coll Cardiol 2011; 57: $1314-1322$.

34. Higo T, Ueda Y, Oyabu J, Okada K, Nishio M, Hirata A, et al. Atherosclerotic and thrombogenic neointima formed over sirolimus drug-eluting stent: An angioscopic study. JACC Cardiovasc Imaging 2009; 2: 616-624.

35. Oyabu J, Ueda Y, Ogasawara N, Okada K, Hirayama A, Kodama K. Angioscopic evaluation of neointima coverage: Sirolimus drug-eluting stent versus bare metal stent. Am Heart J 2006; 152: 1168-1174.

36. Kim JS, Jang IK, Fan C, Kim TH, Park SM, Choi EY, et al. Evaluation in 3 months duration of neointimal coverage after zotarolimuseluting stent implantation by optical coherence tomography: The ENDEAVOR OCT trial. JACC Cardiovasc Interv 2009; 2: 1240 1247.

37. Kim JS, Jang IK, Kim TH, Takano M, Kume T, Hur NW, et al. Optical coherence tomography evaluation of zotarolimus-eluting stents at 9-month follow-up: Comparison with sirolimus-eluting stents. Heart 2009; 95: 1907-1912.

38. Awata M, Nanto S, Uematsu M, Morozumi T, Watanabe T, Onishi T, et al. Angioscopic comparison of neointimal coverage between zotarolimus- and sirolimus-eluting stents. J Am Coll Cardiol 2008; 52: $789-790$.

39. Otagiri K, Tsutsui H, Kumazaki S, Miyashita Y, Aizawa K, Koshikawa $\mathrm{M}$, et al. Early intervention with rosuvastatin decreases the lipid components of the plaque in acute coronary syndrome: Analysis using integrated backscatter IVUS (ELAN study). Circ J 2011; 75: 633641.

40. Finn AV, Kolodgie FD, Harnek J, Guerrero LJ, Acampado E, Tefera $\mathrm{K}$, et al. Differential response of delayed healing and persistent inflammation at sites of overlapping sirolimus- or paclitaxel-eluting stents. Circulation 2005; 112: 270-278.

41. Pires NM, Eefting D, de Vries MR, Quax PH, Jukema JW. Sirolimus and paclitaxel provoke different vascular pathological responses after local delivery in a murine model for restenosis on underlying atherosclerotic arteries. Heart 2007; 93: 922-927.

42. Russo RJ, Silva PD, Teirstein PS, Attubato MJ, Davidson CJ, DeFranco $\mathrm{AC}$, et al. A randomized controlled trial of angiography versus intravascular ultrasound-directed bare-metal coronary stent placement (the AVID Trial). Circ Cardiovasc Interv 2009; 2: 113-123.

43. Oonishi T, Hayabuchi Y, Sakata M, Mori K, Kagami S. Stent placement in the ductus venosus of a neonate with total anomalous pulmonary venous return. J Echocardiogr 2012; 10: 27-29. 